\title{
Parkinson's disease protein DJ-1 regulates ATP synthase protein components to increase neuronal process outgrowth
}

\author{
Rongmin Chen', Han-A Park ${ }^{1,2}$, Nelli Mnatsakanyan', Yulong Niu', Pawel Licznerski', Jing Wu', Paige Miranda', \\ Morven Graham ${ }^{3}$, Jack Tang ${ }^{1}$, Agnita J. W. Boon ${ }^{4}$, Giovanni Cossu', Wim Mandemakers', Vincenzo Bonifati, \\ Peter J. S. Smith $\mathbb{1 0}^{7,8}$, Kambiz N. Alavian ${ }^{8,9}$ and Elizabeth A. Jonas ${ }^{1,8,10}$
}

\begin{abstract}
Familial Parkinson's disease (PD) protein DJ-1 mutations are linked to early onset PD. We have found that DJ-1 binds directly to the $F_{1} F_{O}$ ATP synthase $\beta$ subunit. DJ-1's interaction with the $\beta$ subunit decreased mitochondrial uncoupling and enhanced ATP production efficiency while in contrast mutations in DJ-1 or DJ-1 knockout increased mitochondrial uncoupling, and depolarized neuronal mitochondria. In mesencephalic DJ-1 KO cultures, there was a progressive loss of neuronal process extension. This was ameliorated by a pharmacological reagent, dexpramipexole, that binds to ATP synthase, closing a mitochondrial inner membrane leak and enhancing ATP synthase efficiency. ATP synthase csubunit can form an uncoupling channel; we measured, therefore, ATP synthase $F_{1}$ ( $\beta$ subunit) and c-subunit protein levels. We found that ATP synthase $\beta$ subunit protein level in the DJ-1 KO neurons was approximately half that found in their wild-type counterparts, comprising a severe defect in ATP synthase stoichiometry and unmasking c-subunit. We suggest that DJ-1 enhances dopaminergic cell metabolism and growth by its regulation of ATP synthase protein components.
\end{abstract}

\section{Introduction}

Recent findings in the study of Parkinson's disease (PD) have provided a unifying hypothesis to the apparently disparate functions of the proteins affected by familial genetic mutations. More specifically, many of the proteins encoded by these genes are necessary for normal mitochondrial function and protein trafficking, and show increased expression in tissues with high energy demands and frequent bouts of oxidative stress ${ }^{1-7}$. Rare but important mutations in the gene encoding DJ-1 are found in about $1 \%$ of familial cases of PD. DJ-1 is a peptidase C56 family protein with known and uncharacterized

\footnotetext{
Correspondence: Elizabeth A. Jonas (elizabeth.jonas@yale.edu)

${ }^{1}$ Department of Internal Medicine (Endocrinology), Yale University, New Haven, CT, USA

${ }^{2}$ Department of Human Nutrition and Hospitality Management, University of Alabama, Tuscaloosa, AL, USA

Full list of author information is available at the end of the article.

Edited by P.G. Mastroberardino
}

cellular functions ${ }^{8}$. Despite the creation of a DJ-1 null mouse ${ }^{9}$, the functions of DJ-1 are incompletely understood. DJ-1 mutant animals show increased sensitivity to neuronal toxins, and in different species DJ-1 is required for normal life span, motor function, and neuronal resistance to oxidative damage ${ }^{10-12}$. Defects in DJ-1 alter mitochondrial morphology and function ${ }^{13}$. DJ-1 translocates to mitochondria from the cytosol in response to mitochondrial stress ${ }^{14,15}$, suggesting that DJ-1 may assist Parkin and PINK1 in protein trafficking. DJ-1 also regulates mitochondrial metabolism since DJ-1 mutant cells have impaired ATP production and abnormal respiration $^{11}$. Recent reports have emphasized that DJ-1 mutant mitochondria are sensitive to mitochondrial permeability transition $(\mathrm{PT})^{16}$, one hallmark of cell death, and DJ-1 deficient mitochondria demonstrate abnormally high state 4 respiration, indicative of a leaky mitochondrial inner membrane ${ }^{11}$. 
We recently described that a leak channel that decreases the efficiency of the ATP synthase is located within the csubunit of the membrane-bound portion of the ATP synthase. We and others have suggested that an ATP synthase leak conductance forms a pathological mitochondrial permeability transition pore $(\mathrm{mPTP})^{17-22}$ and that interaction of PD proteins may affect opening of the pore $^{22}$. We described that the purified ATP synthase csubunit ring forms channels in artificial bilayers, and that the channel is regulated by a complex of proteins forming and interacting with the $F_{1}$ or enzymatic portion of the ATP synthase. Reports have shown that regulators within the $F_{1}$ include the subunits $\beta$ and OSCP, the latter of which binds cyclophilin $\mathrm{D}$, an important regulator of mPTP opening and the target of the MPTP inhibitor cyclosporine $A^{20,23}$. We have also shown that the antiapoptotic protein $\mathrm{Bcl}-\mathrm{xL}$, which binds the $\beta$ subunit of $\mathrm{F}_{1}$ ATP synthase, and dexpramipexole (Dex), which binds the $\mathrm{OSCP} / \mathrm{b}$ subunits, enhance the relative closure of the leak channel ${ }^{24-26}$.

A normal function of the leak channel is to adjust the level of uncoupling of oxidative phosphorylation to enhance or decrease metabolic efficiency. We now find that DJ-1 binds to the ATP synthase $\beta$ subunit, influencing the probability of closure of the ATP synthase leak channel. DJ-1 also regulates the level of $\beta$ subunit protein in mitochondria, ensuring the proper stoichiometric relationship of $\beta$ subunit to c-subunit. This relationship is required for normal ATP synthase function. Thus, we find that DJ-1 regulates normal ATP synthase function and stoichiometry to enhance growth of neuronal processes, protect cells from death, and promote oxidative metabolic efficiency.

\section{Results}

DJ-1 is localized to the ATP synthasome and interacts directly with ATP synthase $\beta$ subunit

In looking for ATP synthase binding partners or modulators of the ATP synthase leak channel, we isolated submitochondrial vesicles (SMVs) that are relatively enriched in ATP synthase ${ }^{24,27,28}$. Liquid chromatography mass spectrometry analysis of the proteins from SMVs revealed the presence of DJ-1. To confirm this, we performed immunoblots of SMVs with anti-DJ-1 antibodies and demonstrated the presence of DJ-1 (Fig. 1a). In previous reports, we showed that the anti-cell-death protein $\mathrm{Bcl}-\mathrm{xL}$ was bound to the $\beta$ subunit of the ATP synthase and that it regulates inner mitochondrial membrane leak to enhance cellular metabolism ${ }^{24,26}$. To determine if DJ-1 were also bound to the $\beta$ subunit, we immunoprecipitated FLAG-tagged DJ-1 from HEK-293T cells using beads conjugated to an anti-FLAG antibody (Fig. S1a); this revealed colocalization of DJ-1 and the $\beta$ subunit (Fig. 1b).
Since the interaction was robust, we tested the interaction of purified WT DJ-1 with ATP synthase $\beta$ subunit in vitro (Fig. 1c). Purified recombinant FLAG-tagged ATP synthase $\beta$ subunit protein was incubated with purified recombinant DJ-1, and the mixture was immunoprecipitated with anti-DJ-1 antibodies. This demonstrated that the two proteins bind to each other directly and do not require other binding partners for their interaction. We also found that DJ-1 coimmunoprecipitates with Bcl-xL (Fig. S1b), suggesting that the two proteins could regulate cellular metabolism by working together in a complex. To test if $\mathrm{DJ}-1$, like $\mathrm{Bcl}-\mathrm{xL}$, is present in mitochondrial inner membranes in the brain, we performed immunoelectron microscopy. We found that DJ-1 localizes both to outer membrane and to inner membrane cristae in 9/12 micrographs.

\section{Mutant DJ-1 proteins fail to enhance ATP synthase function}

ATP synthase produces ATP in mitochondria. To study the function of WT DJ-1 and loss of function DJ-1 mutations in mitochondrial ATP production, we expressed DNA constructs for known PD-associated DJ-1 mutations $\left(\mathrm{M} 3=\mathrm{A} 104 \mathrm{~T} ; \mathrm{M} 4=\mathrm{D} 149 \mathrm{~A} ; \mathrm{M} 5=\mathrm{L} 166 \mathrm{P} ;{ }^{12}\right.$ and for WT DJ-1 in HEK 293T cells; we also purified the proteins). Surprisingly we found that all mutant DJ-1 proteins bound effectively to both the ATP synthase $\beta$ subunit and to Bcl-xL (Fig. 1b and S1b). We also noted that the C-terminal 60 amino acid deletion in DJ-1 does not affect its binding to the ATP synthase $\beta$ subunit (Fig S1c). Therefore, we determined if binding was sufficient to confer normal ATP synthase enzymatic function. To assess if DJ-1 binding to the $\beta$ subunit were required for normal inner membrane $\mathrm{H}^{+}$ion sequestration, we performed a 9-amino-6-chloro-2-methoxyacridine (ACMA) assay. SMVs purified from rat brain were incubated with the $\mathrm{H}^{+}$fluorescent indicator ACMA and exposed to $\mathrm{ATP}^{24,29}$. ATP hydrolysis in this assay produces sequestration of $\mathrm{H}^{+}$ions into the lumen of the SMVs, and an accompanying decrease in bath fluorescence of the SMVexcluded $\mathrm{H}^{+}$indicator. The steady state response to ATP was enhanced by the addition of WT DJ-1 protein to the bath. In contrast, the addition of mutant proteins failed to enhance the steady state level of $\mathrm{H}^{+}$ion sequestration (Fig. 1a and S1d). DJ-1 deficient SMVs isolated from DJ$1^{-l-}$ mouse brain also failed to sequester $\mathrm{H}^{+}$normally in response to ATP hydrolysis (Fig. 1e).

An improvement in $\mathrm{H}^{+}$sequestration in the ACMA assay may indicate an increase in ATPase rate, a decrease in inner membrane leak or both. To differentiate between these effects, we measured ATPase rate and leak directly using different techniques. ATPase activity was assessed in an ATP regenerating system where ATP concentration 


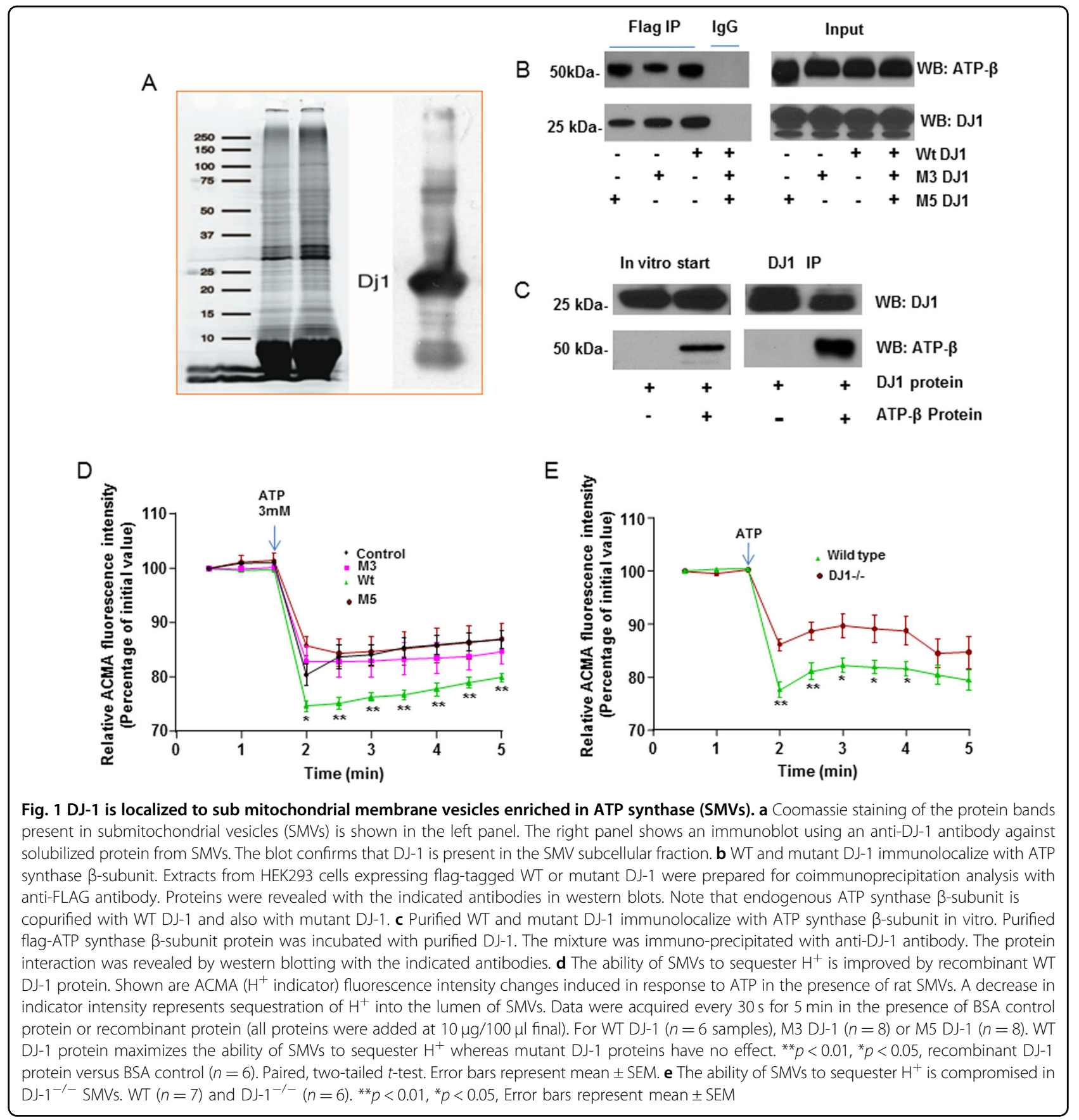

is not rate-limiting (Fig. 2a and S2). WT DJ-1 protein markedly enhanced enzymatic rate while mutant forms of DJ-1 protein did not enhance ATPase activity over control. To test if DJ-1 or mutants regulated the availability of ATP, ATP levels were measured in DJ-1 overexpressing HEK $293 \mathrm{~T}$ cells and primary midbrain cultures. ATP levels were found to be significantly higher when WT DJ1, but not mutant DJ-1, was overexpressed (Fig. 2b). ATP levels were lower in DJ-1 $1^{-1-}$ midbrain cultures compared to those of WT controls (Fig. 2c).

\section{Mutant DJ-1 proteins fail to close an inner mitochondrial membrane leak}

The ATPase assays suggested that WT DJ-1 enhances ATPase enzymatic rate; these assays, however, do not specifically test whether DJ-1 prevents mitochondrial inner membrane leak. Therefore, to determine whether the effect of DJ-1 on $\mathrm{H}^{+}$ion sequestration rate was due to a decrease in the inner membrane leak produced by DJ-1, we performed patch clamp recordings of rat brain SMVs. WT control recordings showed significantly higher ion 

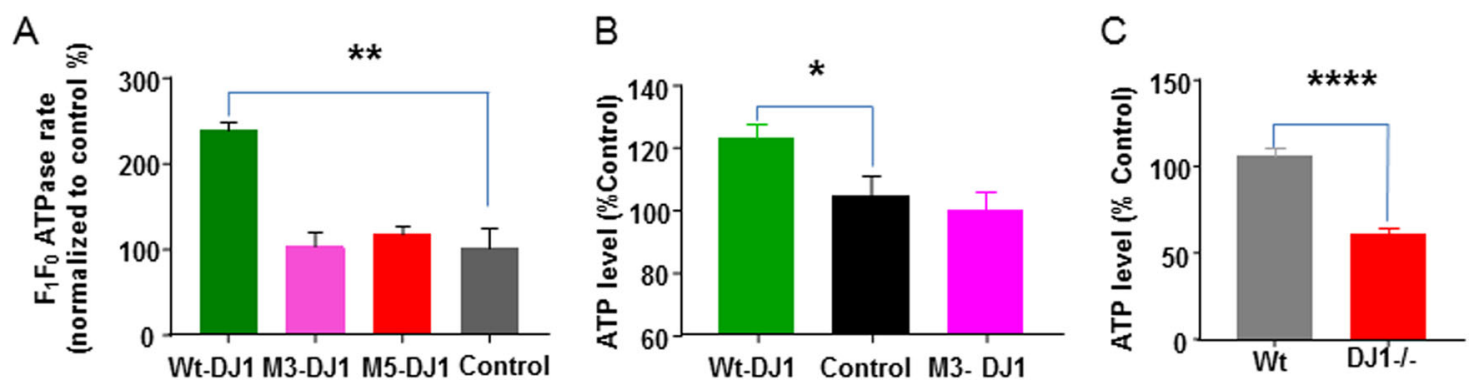

Fig. 2 DJ-1 is required for optimal efficiency of ATP synthase enzyme activity. a Recombinant WT DJ-1 protein, but not M3 and M5 mutant proteins, increases ATP hydrolysis enzymatic rate in an assay where ATP level is not rate-limiting (see methods). Recombinant proteins were added to the wells during the assay ( $N=4$ for each assay group; ${ }^{* *} p<0.01$. Error bars represent mean $\pm S E M$; unpaired, two-tailed t-test.). $\mathbf{b}$ WT DJ-1, but not mutant DJ-1 (M3), increases endogenous ATP levels when overexpressed in HEK293 cells $\left({ }^{*} p<0.05, n=7\right.$, Error bars represent mean \pm SEM). c The endogenous ATP level in DJ-1 ${ }^{-1-}$ midbrain culture is significantly lower than that of WT midbrain cultures. Mean \pm SEM is shown. $N=72$ wells from three independent paired primary cultures $\left({ }^{* * *} p<0.0001\right)$

channel conductance at all voltages recorded compared to SMVs exposed to recombinant WT DJ-1 protein, suggesting that addition of extra, exogenous DJ-1 further decreases leak channel activity even in WT SMVs (Fig. 3a-c). Conductance was further decreased by addition of ATP to the bath, consistent with previously reported findings ${ }^{17,24}$. WT mouse SMVs also responded with a rapid decrease in conductance after the addition of ATP, while DJ-1 ${ }^{-1-}$ SMVs failed to respond to ATP, suggesting that the ability of ATP to alter the inner membrane ion channel conductance is dependent on the presence of DJ-1 (Fig. 3d). To test this finding further, we recorded DJ-1 $1^{-1-}$ SMVs and added either recombinant WT or mutant DJ-1 protein to the bath during recordings. The addition of WT DJ-1 decreased conductance of the patch in both WT mouse brain SMVs and DJ-1 ${ }^{-1-}$ SMVs, so much so that there was little additional effect of ATP on inner membrane conductance, whereas the addition of mutant protein samples had no effect (Fig. 3e, f). ATP also failed to decrease conductance in DJ-1 ${ }^{-1-}$ SMVs exposed only to mutant DJ-1 protein. This latter finding confirmed that WT DJ-1 and ATP are required for maximally decreasing leak conductance (Fig. 3e, f).

The decrease in inner membrane leak and the increase in ATPase enzyme activity may prevent a loss of inner membrane potential caused by metabolic activities or by a pathological inner membrane leak. In support of this, mitochondrial membrane potential was found to be relatively hyperpolarized in normal WT neurons compared to neurons lacking DJ-1 (Fig. 4a, b and S3). Hyperpolarization of mitochondrial membrane potential could accompany an increase in efficiency of ATP production associated with less substrate uptake per ATP molecule produced $^{24}$. We had already found an increase in ATP levels in the neuronal cultures (Fig. 2). We therefore measured oxygen consumption in single neurons. Single neuron oxygen sensors deliver real time oxygen flux detection with high spatial resolution ${ }^{24,25,30-32}$, allowing the interrogation of single cells using a 1-2 $\mu \mathrm{m}$ diameter tip held within $1 \mu \mathrm{m}$ of the cell surface. Using the sensor, we found that oxygen consumption by single WT neurons was significantly lower than that in neurons depleted of DJ-1 by shRNA (Fig. 4c, d), suggesting that the low inner membrane leak increases ATP production per unit oxygen consumed.

\section{DJ-1 enhances growth of neuronal processes}

Neurons experiencing metabolic derangements may be placed at risk for degeneration, developmental defects, or defects in growth or branching of neuritic processes. We found that during 4 weeks in culture, neurites of midbrain dopaminergic neurons (stained with anti- $\mathrm{TH}$ antibody; Fig. S4a) isolated from $\mathrm{DJ}-1^{-1-}$ mice failed to reach the same length as WT neurites and there was a decrease of neurite numbers at initiation sites on the neuronal somata (Fig. $5 \mathrm{a}-\mathrm{c}$ ). The number of branch points of each neurite was also reduced (Fig. 5d). These growth deficiencies were associated with chronic cell death in the cultures as measured by lactate dehydrogenase (LDH) release into the medium by the third week (Fig. 5e). Enhanced death rate was most likely related to the abnormal metabolism produced by the absence of DJ-1. To determine if closing the inner membrane leak would improve functional outcomes, we treated with the pharmacological reagent Dex, that we have shown previously to decrease inner mitochondrial membrane conductance and ameliorate cell death $^{25,33,34}$. Dex effectively rescued the loss of neurites at the cell soma in dopaminergic neurons cultured chronically over 3-4 weeks, although neurite length was not significantly improved (Fig. $5 \mathrm{f}$ and S4b), suggesting that attempted pharmacological improvement in inner membrane coupling was only partially successful in preventing loss of neurites and neurite growth in DJ-1 deficient cultures. 


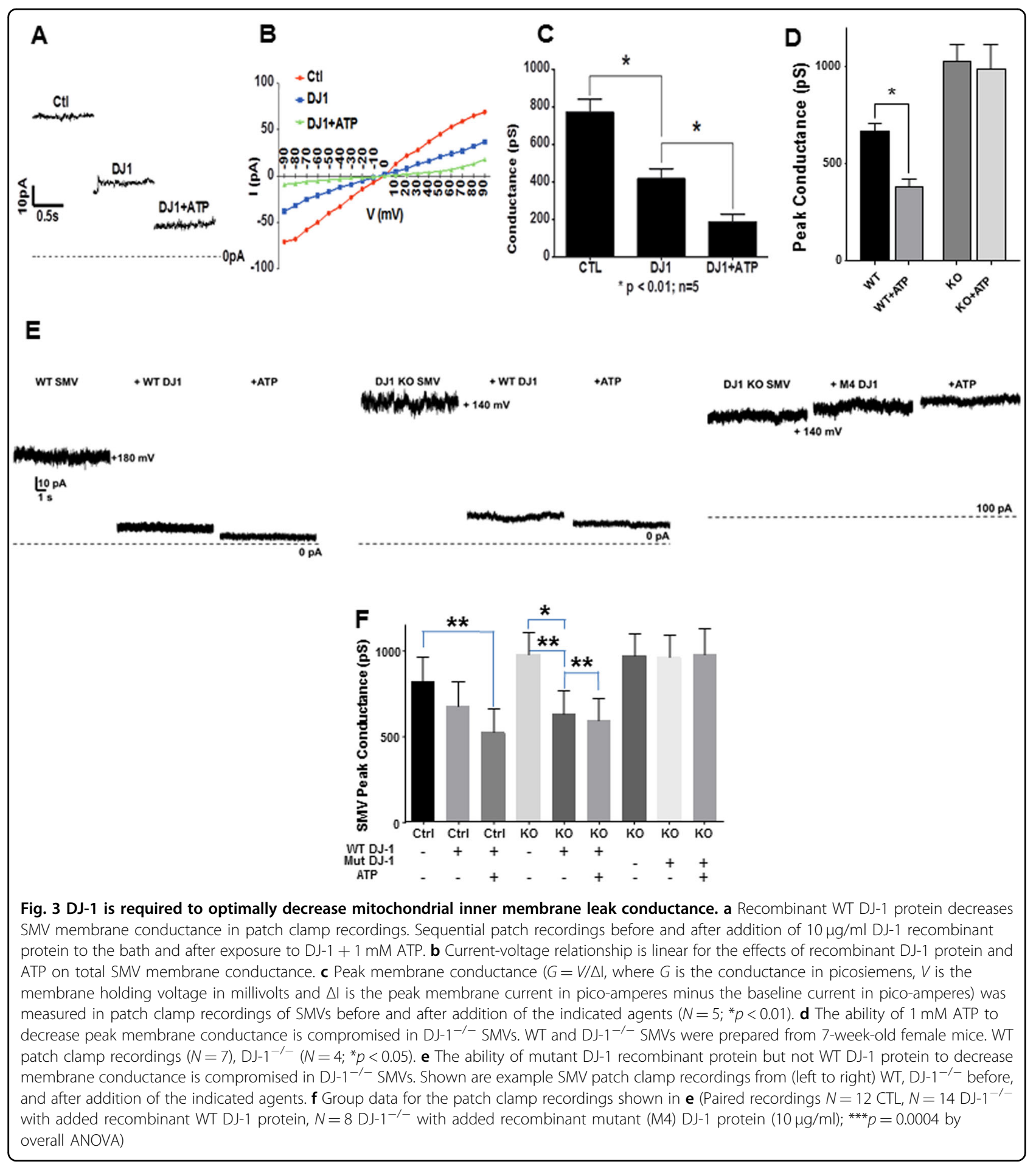

We had suggested previously that the ATP synthase csubunit leak channel is gated by interaction with components of the $F_{1}{ }^{17}$. If some $F_{1}$ components were lost in the DJ-1 deficient cultures, this would eliminate the binding site for common reagents used to enhance inner membrane coupling like Dex ${ }^{20,23}$. To determine if the metabolic and growth defects produced by the lack of DJ-
1 were related to relative loss of $\mathrm{F}_{1}$ components compared to $c$-subunit, we examined $c$-subunit and $\beta$ subunit mRNA in mesencephalic cell culture by qPCR. The main isoform of c-subunit in neurons, ATP5G1 mRNA, was significantly increased in DJ-1 deficient neurons compared to WT neurons, while levels of $\beta$ subunit mRNA were unchanged (Fig. 5g). This was also the case in aging 
A
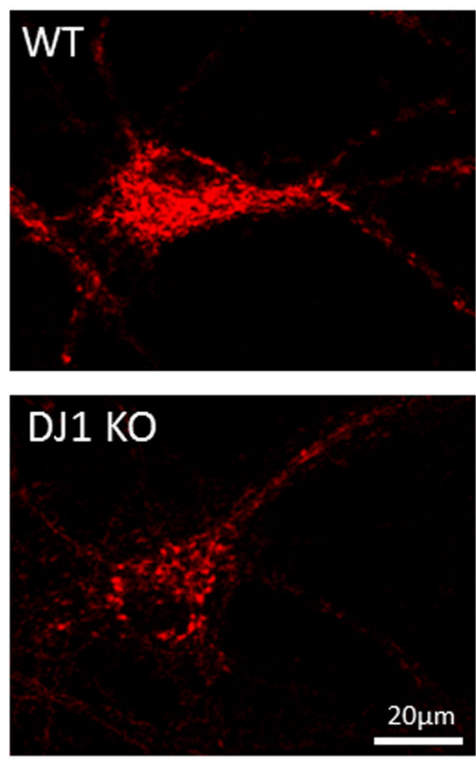

C

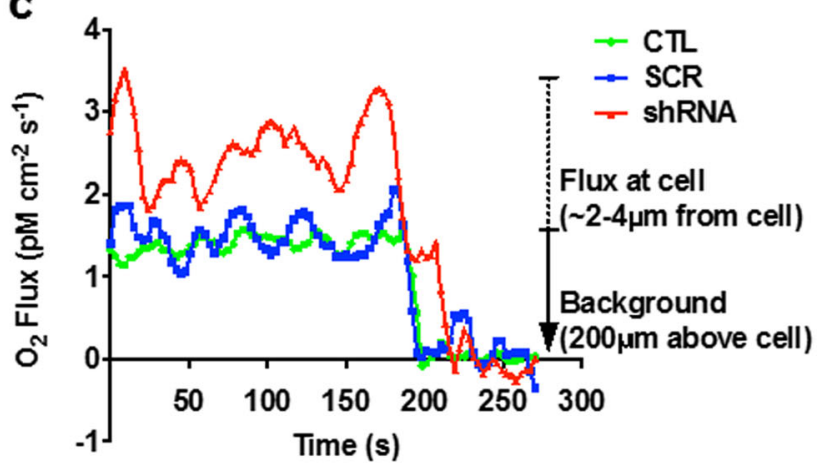

B

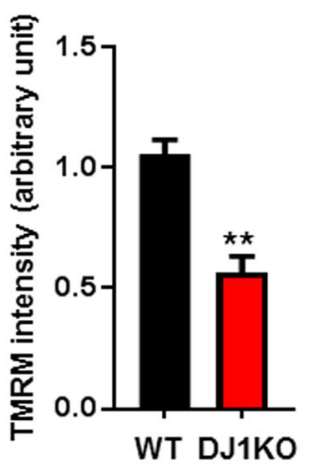

$\mathrm{N}=3$ paired independent cultures

Fig. $4 \mathrm{DJ}-1^{-1-}$ neurons have reduced mitochondrial membrane potential. a Representative images of mitochondrial membrane potential indicator TMRM of WT and DJ-1 $1^{-1-}$ neurons. $\mathbf{b}$ Group data for images shown in $\mathbf{a}$. TMRM fluorescence intensity was calculated by drawing a large region of interest that encompassed the entire soma ( $n=45 \mathrm{WT}$ neurons and $n=43 \mathrm{DJ}^{-1-}$ neurons from three independent paired midbrain cultures, $\left.{ }^{* *} p=0.005964\right)$. c Presence of DJ-1 reduces oxygen consumption of single hippocampal neurons. Panel shows representative traces of single neuron oxygen electrode recordings performed in WT neurons or WT neurons expressing scrambled or DJ-1 shRNA. d Group data of peak oxygen consumption for single neurons expressing the indicated constructs $\left(N=7-11\right.$ cells each; $\left.{ }^{*} p=0.0136\right)$

mouse brain where ATP5G1 (c-subunit) mRNA was again found to be significantly increased in DJ-1 deficient brain tissue while ATP5B ( $\beta$ subunit) mRNA was reduced (Fig. $5 \mathrm{~h})$. Immunoblots of aging brain mitochondria revealed that $\beta$ subunit protein level was substantially reduced, while c-subunit protein level was relatively unaffected (Fig. 5i), suggesting a change in stoichiometry within the ATP synthase proteome that would predispose mitochondria to a leaky inner membrane.

\section{Discussion}

DJ-1 binds to the $\beta$ subunit to affect ATP synthase enzyme function and inner mitochondrial membrane permeability

We show in isolated neurons, HEK cells and isolated mitochondria that mutation or loss of DJ-1 cause a decrease in ATP synthase enzymatic rate, a decrease in cellular ATP levels, loss of mitochondrial inner membrane potential and development of a large leak in the mitochondrial inner membrane. These findings are accompanied by decreased protein levels of ATP synthase $\beta$ subunit and a decrease in the ratio of $\beta$ subunit mRNA and protein to those of c-subunit. We suggest that this promotes ATP synthase leak channel opening. Our previous studies have shown that the c-subunit can form a large conductance channel under conditions of excitotoxic stress ${ }^{17,35}$, suggesting that the membrane portion of the ATP synthase constitutes the main pore-forming unit of the mPTP. We show here in patch clamp recordings that DJ-1 decreases the propensity for the mitochondrial inner membrane leak to open. Our findings of inner 


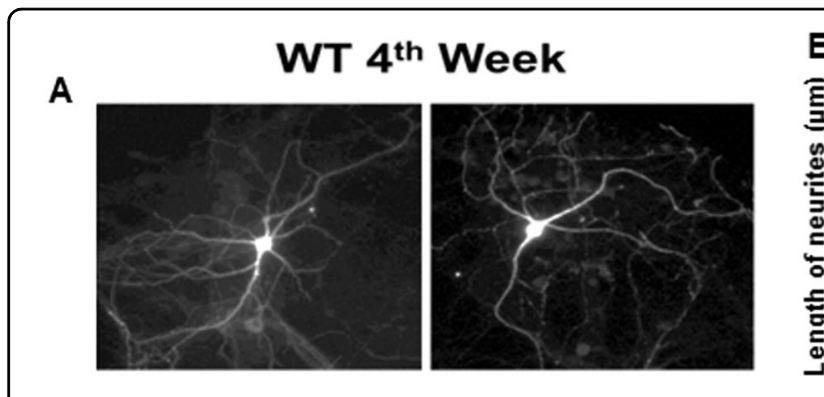

DJ1 KO $4^{\text {th }}$ Week
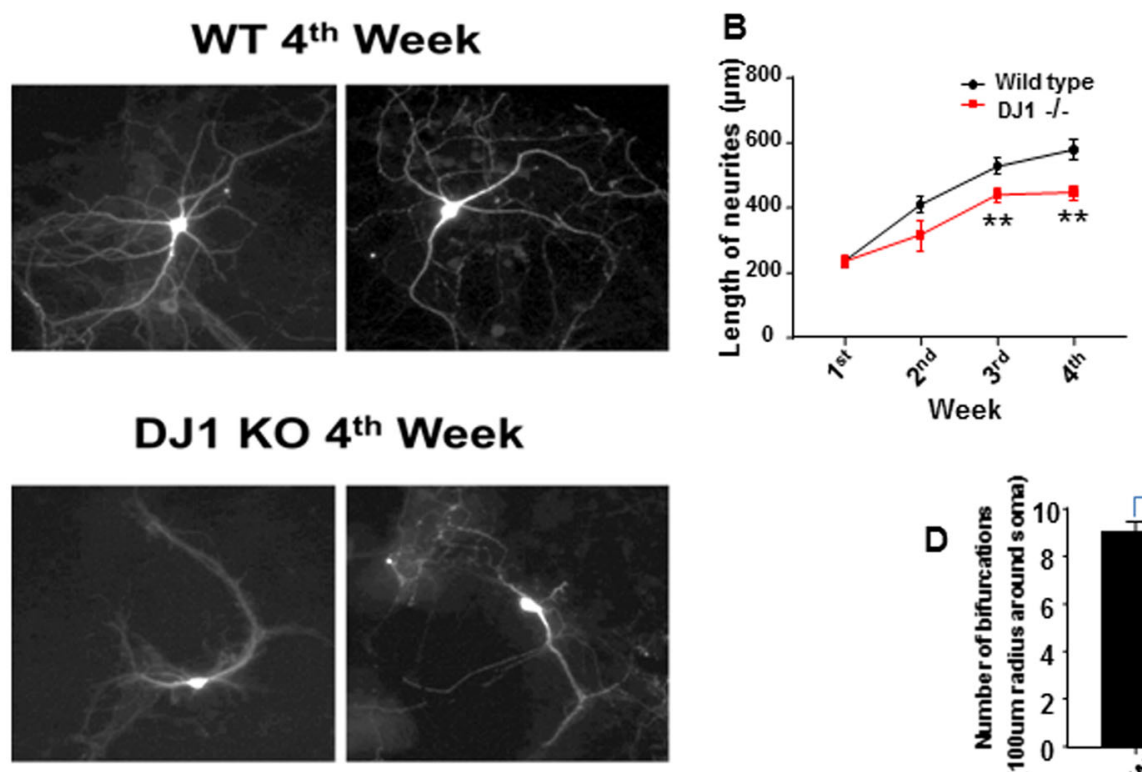

C
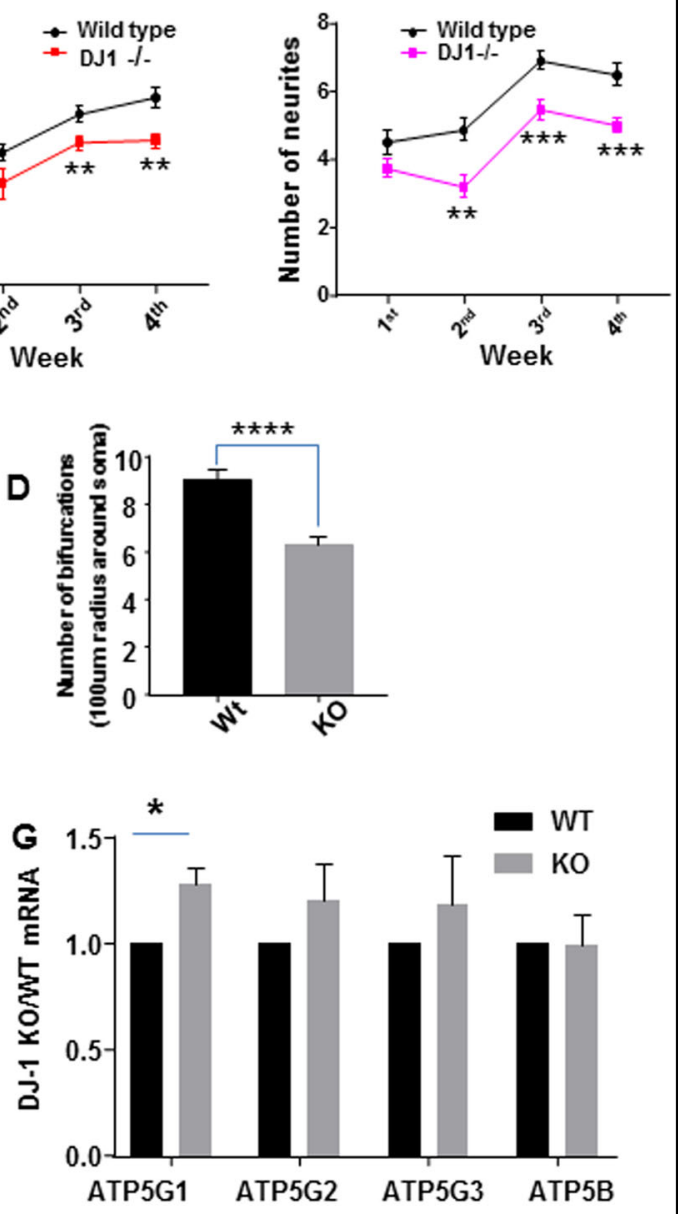

$\mathrm{KO} \mathrm{KO}+\mathrm{Dex}$

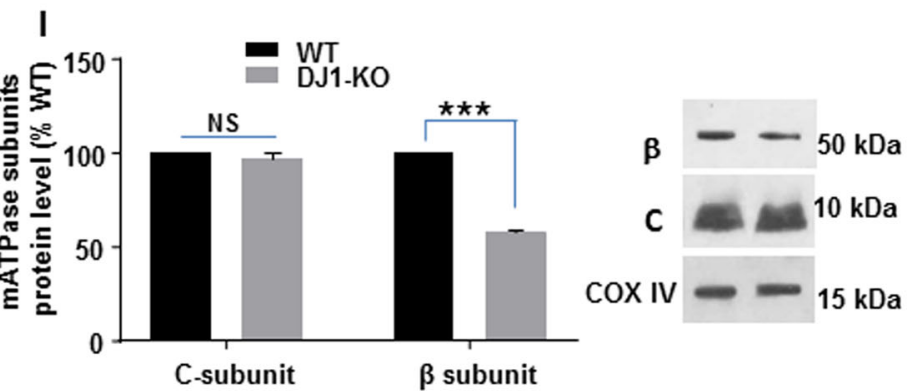

Fig. 5 DJ-1 depletion inhibits neurite outgrowth of dopaminergic neurons. a Representative tyrosine hydroxylase (TH) antibody-stained neurons. b The length of neurites at four different time points in vitro (First week: WT ( $n=26 \mathrm{TH}+$ neurons), KO $(n=20)$; second week: WT ( $n=25)$, $\mathrm{KO}(n=16)$; third week: WT $(n=41), \mathrm{KO}(n=32),{ }^{* *} p=0.0091$; fourth week: WT $\left.(n=22), \mathrm{KO}(n=32),{ }^{* *} p=0.0012\right)$. c The number of neurites extending directly from the soma at four different time points in vitro (First week: WT $(n=26), \mathrm{KO}(n=20)$; second week: WT $(n=25), \mathrm{KO}(n=16)$, ${ }^{* *} p$ $=0.0022$; third week: WT $(n=41), \mathrm{KO}(n=32)$, ${ }^{* * *} p=0.00073$; fourth week: WT $(n=22), \mathrm{KO}(n=32)$, $\left.{ }^{* *} p=0.00026\right)$. $\mathbf{d}$ The number of bifurcations at the third week ( $n=20 \mathrm{WT} \mathrm{TH}+$ neurons and $n=20 \mathrm{DJ}-1^{-1-} \mathrm{TH}+$ neurons, $\left.{ }^{* * * *} p<0.0001\right)$. e LDH release (into the medium) at the third week of culture ( $n=3$ cultures, ${ }^{*} p=0.0052$ ). $\mathbf{f}$ Neurite outgrowth of dopaminergic neurons is improved by $30 \mu \mathrm{M}$ Dex. Number of neurites extending directly from the soma at the third week (Left). Length of neurites at the third week (Right). The length of untreated KO dopaminergic neurites and the number of untreated $\mathrm{KO}$ dopaminergic neurites are set as 100\%, Dex treatment affects the number of neurites arising from the soma. Mean \pm SEM ( $n$ $=3$ independent midbrain cultures, ${ }^{*} p=0.018$ for neurite number, at least 10 cells counted per group in each culture). $\mathbf{g}$ ATP synthase c-subunit $5 \mathrm{G} 1$ mRNA is increased in DJ-1 ${ }^{-1-}$ compared to WT midbrain cultures. The mRNA level in WT cells was set as one, mean \pm SEM ( $n=3$ independent cultures, ${ }^{*} p=0.027$ for ATP5G1). $\mathbf{h}$ mRNA expression of ATP5G and ATP5B in 7-month-old mouse midbrain tissue. The data are quantified by qRT-PCR. The mRNA level in WT was set as one, mean \pm SEM $\left(n=3\right.$ for WT mouse and $n=3$ for DJ- $1^{-1-}$ mouse, ${ }^{*} P=0.0108$ for ATP5G1 and ${ }^{* *} p=0.0027$ for ATP5B). i Mitochondrial protein expression of ATPase c-subunit is unchanged (Left), but mitochondrial protein expression of ATPase $\beta$ subunit is decreased in DJ-1 KO brain (Right) of 7-8-month-old animals. Protein level in WT was set as $100 \%{ }^{* *} p=0.0002$ for ATP synthase $\beta$ subunit level 
membrane depolarization in the absence of DJ-1 are supported by previous studies in the field that have suggested that loss of DJ-1 leads to inner membrane depolarization, $\mathrm{PT}^{16}$, and oxidative stress ${ }^{36}$. In a role for DJ-1 that has never been previously suggested, we find that the binding of DJ- 1 to the $\beta$ subunit increases ATPase activity, the level of ATP in cells, and decreases oxygen uptake, enhancing inner membrane coupling, and metabolic efficiency of oxidative phosphorylation. In addition, the correct stoichiometry between the $\mathrm{F}_{1}$ and c-subunit, regulated by the presence of WT DJ-1, appears to be required for full efficiency in ATP synthase function.

\section{DJ-1 enhances $\beta$ subunit amount in mitochondria}

DJ-1 has been described to have multiple functions, particularly as a modulator of oxidative stress, in part by directly protecting cells from toxic glycolytic products by becoming oxidized itself ${ }^{37}$. DJ-1 also acts as a coactivator of various transcription factors including the androgen receptor, p53, PSF, Nrf2, SREBP, and RREB1 and the LDL receptor $^{38-40}$. In this role, DJ-1 upregulates signaling pathways involved in the expression of genes that combat oxidative stress and it enhances pathways regulating cell survival and growth ${ }^{10}$.

Recent reports have also suggested that DJ-1 acts as a chaperone or chaperone regulator; increasing DJ-1 expression increases levels of Hsp70, preventing aggregation of alpha synuclein ${ }^{41}$. WT as well as mutant DJ-1 have been found to associate with Hsp70, mitochondrial Hsp70/Grp75 (mortalin), and the cochaperone C-terminus of Hsp70 interacting protein. DJ-1 translocates into mitochondria upon oxidative stress accompanied by other chaperones suggesting that it works together with these chaperones ${ }^{42}$. Further evidence of its chaperone function is that overexpression of DJ-1 protects neurons from alpha synuclein aggregation by increasing the expression of LAMP-2A, suggesting that chaperone mediated autophagic function is enhanced by DJ-1 ${ }^{43,44}$. Nevertheless, despite known mitochondrial localization by DJ-1, chaperone function of DJ-1 for ATP synthase subunits, as suggested by our study, has not been previously reported. We now find that DJ-1 is necessary for the proper complement of $\beta$ subunit protein in mitochondria, but whether DJ-1 acts at the transcriptional level, protects $\beta$ subunit mRNA, helps with translation of $\beta$ subunit mRNA and/or helps translocate $\beta$ subunit into the mitochondria or all of the above remains to be fully studied. We suggest that these functions may be key to regulate ATP synthase activity under stressful conditions or during aging.

\section{$\beta$ subunit of ATP synthase forms a hub for regulation of metabolism}

The ATP synthase $\beta$ subunit and other components of the enzymatic $F_{1}$ portion of the synthase respond to, and regulate, metabolic flexibility. ATP synthase $F_{1}$ subunits work in a complex with regulators, including antiapoptotic proteins such as $\mathrm{Bcl}-\mathrm{xL}$. In mouse neurons with decreased levels of $F_{1}$ components including OSCP and the $\beta$ subunit, mitochondrial membrane potential is depolarized, accompanied by increased oxidative stress, early PT, low ATP levels and decreased synaptic function $^{45}$. The same group also found low levels of $F_{1}$ components in Alzheimer's-diseased brains. We have previously reported that the anti-death protein Bcl-xL binds to the $\beta$ subunit of the ATP synthase and enhances mitochondrial ATP production efficiency ${ }^{24,26}$. This leads to enhanced synapse formation and enhanced synaptic transmission $^{46,47}$ as well as increased localization of mitochondria to synaptic sites through activation of mitochondrial fission ${ }^{47,48}$. Our current study suggests that in addition to its own effects on $\beta$ subunit and ATP synthase function, DJ-1 also binds to $\mathrm{Bcl}-\mathrm{xL}$, perhaps enhancing Bcl-xL's activity to increase ATP production, mitochondrial positioning, and neurite extension.

\section{Cell and axonal growth are affected by loss of DJ-1}

Evidence suggests that normal neuronal development is dependent upon kinase pathways that assist in mitochondrial positioning at axonal branch points ${ }^{49}$. Mitochondrial morphological changes consistent with a loss of fusion/fission have been observed in neurons and patient cells with DJ-1 deficiency ${ }^{50}$. A recent study shows that Parkin KO neurons grown on astrocyte monolayers of the same genotype have a markedly decreased axonal arborization, increased basal oxygen consumption rate, and decreased ATP production, consistent with uncoupling ${ }^{51}$. These neurons also have reduced dopamine transporter, as a possible mechanism for decreased dopaminergic function in parkin deficient animals. Although no change in axonal arborization was found for DJ-1 neurons in that study, the authors acknowledged that increasing time in the culture might be necessary to detect changes. Indeed, our findings show that growth of neuronal processes over 4 weeks in culture is dependent upon DJ-1, and we show that marked slowing of growth, with accompanying neuronal loss, occurs over this time in DJ-1 lacking cells. Decreased neuronal process outgrowth was also observed in an animal model of DJ-1 loss on a C57B6 background. In that report, in the SNc, marked tyrosine hydroxylase $(\mathrm{TH})^{+}$neuronal loss was demonstrated and neurons clearly had shortened neuronal processes and process beading, with age dependent loss of process length and sprouting, decrease in DA-synthesizing $\mathrm{TH}^{+}$striatal terminals and motor dysfunction ${ }^{52}$. In the DJ-1 KO neurons, our current findings suggest partial rescue of the lack of growth of the neurites with Dex, a reagent that we have shown increases mitochondrial inner membrane coupling by binding to ATP synthase $F_{1}$. We found that 
the effect of Dex was only partial, perhaps because, in the absence of the full complement of $\beta$ subunit, Dex is unable to fully close the leak ${ }^{53}$.

Our data suggest that DJ-1 is required for creating the normal stoichiometry of the ATP synthase and for assisting with positioning of ATP synthase $\beta$ subunit to fully close the mitochondrial inner membrane leak. Therapeutic potential would therefore be provided by agents that enhance or mimic the functions of DJ-1 to position the $\beta$ subunit, to synthesize $\beta$ subunit, and to translocate $\beta$ subunit into the inner membrane. Our study suggests a role for DJ-1 in transcriptional/translational regulation and chaperone function directed specifically at the ATP synthase $\beta$ subunit.

\section{Methods}

Animals

WT mice and DJ-1 $1^{-1-}$ mice were from The Jackson Laboratory (C57BL/6, Stock no: 000664; B6.CgPark $7^{\mathrm{tm} 1 S h n} / J$, Stock no: 006577) and the original DJ-1 ${ }^{-1-}$ mouse was previously described ${ }^{54}$. Sprague-Dawley rats were from Charles River. All animals were maintained in a climate-controlled room and kept on a 12:12-h light-dark cycle with food and water available. All procedures were performed in accordance with the NIH Guide for the Care and Use of Laboratory Animals and approved by Yale University's Institutional Animal Care and Use Committee (IACUC).

\section{Recombinant protein expression and purification}

The human DJ-1 and ATP synthase $\beta$ (ATP5B) constructs with Myc and DDK (Flag) tags on the C-terminus were from OriGene Technologies. Five different DJ-1 mutations were described in a previous study ${ }^{12}$, M1 (M26I), M2 (E64D), M3 (A104T), M4 (D149A), and M5 (L166P) were made from the original DJ-1 construct using a Quick-change Site-Directed Mutagenesis Kit (Agilent Technologies). HEK293 cells were cultured in $15 \mathrm{~cm}$ cell culture dishes in high glucose DMEM (10\% v/v FBS, $100 \mathrm{U} / \mathrm{ml}$ penicillin, $100 \mu \mathrm{g} / \mathrm{ml}$ streptomycin). All the plasmid transfections were performed with Lipofectamine 2000 (Invitrogen). Recombinant proteins were purified using the EZview Red ANTI-FLAG M2 Affinity Gel (Sigma, F2426), according to the manufacturer's protocol. The expression was verified by western blot analysis according to previously published protocols ${ }^{17,55}$.

\section{Western blot, Coimmunoprecipitation, and in vitro IP}

Cells were lysed at $4{ }^{\circ} \mathrm{C}$ in extraction buffer $(0.1 \mathrm{M} \mathrm{NaCl}$, $20 \mathrm{mM}$ HEPES pH 7.5, $1 \mathrm{mM}$ EDTA, $5 \mathrm{mM} \mathrm{NaF}, 1 \mathrm{mM}$ dithiothreitol, $0.3 \%$ Triton X-100, $5 \%$ glycerol, $0.25 \mathrm{mM}$ phenylmethylsulfonyl fluoride, and complete protease inhibitor cocktail and phosphatase inhibitors). Homogenates were cleared by centrifugation $(12,000 \mathrm{~g}, 10 \mathrm{~min})$.
Coimmunoprecipitation was performed according to previous techniques ${ }^{55}$. In vitro IP was performed using purified recombinant proteins instead of the cell lysate. The antibodies used in the study were as follows: AntiMyc (Cell Signaling, \#2276); Anti-DDK (Origene, TA50011); Anti-DJ-1(Santa Cruz, sc27006 and sc32874; Cell Signaling, 2134S); Anti- $\beta$-Actin, \#4970); AntiGAPDH (Santa Cruz, sc-32233); Anti-mATP5G1/2/3 (abcam, ab180149); Anti-ATPB (abcam, ab14730), and Anti-Bcl-xL(Cell Signaling, \#2764); Anti-Puromycin (3RH11) (Kerafast, EQ0001).

\section{Isolation of mitochondria}

Mitochondria were isolated from human fibroblasts following QproteomeTM Mitochondria isolation kit manual (Qiagen, Cat.37612). In brief, cell pellets were harvested and washed once using $0.9 \% \mathrm{NaCl}$, then cells were lysed in buffer and the lysate was centrifuged at $1000 \mathrm{~g}$ for $10 \mathrm{~min}$. The supernatant was saved as the cytosolic protein sample. Cell disruption was carried out using a $1 \mathrm{ml}$ syringe with a blunt-ended needle in disruption buffer (provided in the kit) times ${ }^{10}$. The lysate supernatants were saved after centrifugation at $1000 \mathrm{~g}$ for $10 \mathrm{~min}$. The pellet disruption step was repeated and the supernatants were combined. The mitochondrial pellets were collected after supernatants were centrifuged at $6000 \mathrm{~g}$ for $10 \mathrm{~min}$. After washing with storage buffer and centrifuging at $6000 \mathrm{~g}$ for $20 \mathrm{~min}$, the mitochondrial pellets were resuspended in lysis buffer as the mitochondrial protein sample.

\section{Isolation of SMVs from rodent brain}

Rodent brain tissue (without cerebellum) was finely minced and homogenized in ice-cold isolation buffer [250 mM sucrose, $20 \mathrm{mM}$ Hepes (pH 7.2), $1 \mathrm{mM}$ EDTA, and $0.5 \% \mathrm{BSA}]$. After a centrifugation at $1500 \mathrm{~g}$ to pellet nuclear material, the supernatants were centrifuged at high-speed $(16,000 \times g)$ for $10 \mathrm{~min}$ at $4{ }^{\circ} \mathrm{C}$. The crude pellets were resuspended in isolation buffer and a pressure of 1200 Psi was applied for $10 \mathrm{~min}$, followed by rapid decompression. The pure mitochondrial fraction was then pelleted in a ficoll density gradient by centrifugation and washed with isolation buffer. Nonionic detergents (digitonin and Lubrol-PX) were used to further solubilize and stabilize membrane-bound protein complexes, and the submitochondrial SMVs were isolated by a final $2 \mathrm{~h}$ ultracentrifuge. Freshly prepared SMVs were quantitated using the Bradford protein assay and used in ACMA experiments and in the ATP regeneration system.

\section{ACMA assay}

ACMA (Sigma A5806) fluorescence quenching was measured according to previously published methods ${ }^{24}$ with some modifications. In brief, $2 \mu \mathrm{M}$ ACMA, $50 \mu \mathrm{g}$ 
isolated rat brain $\mathrm{F}_{1} \mathrm{~F}_{\mathrm{O}}$ ATPase vesicles, and equal amount of either WT DJ-1 or mutant DJ-1 protein was used in each reaction. $F_{1} F_{O}$ ATPase vesicle suspensions were measured at $490 \mathrm{~nm}$ using a PerkinElmer VICTOR3 multilabel plate reader. Similar assays were performed on WT and DJ-1 ${ }^{-1-}$ mice brains.

\section{Neuronal culture and studies of cultured neurons}

WT and DJ-1 ${ }^{-1-}$ midbrain (or cortical) cultures were prepared from embryos isolated from a pregnant female mouse at day-13 post coitum. Cells were dissociated and seeded on Poly-Lysine pre-treated culture dishes with neurobasal medium, supplemented with B-27, glutamine, antibiotics and 5\% FBS (Invitrogen GIBCO Life Technologies, Carlsbad, CA, USA). After $2 \mathrm{hr}$ incubation, primary cultures were maintained in neurobasal medium without $\mathrm{FBS}$ in $5 \% \mathrm{CO} 2$ incubator at $37^{\circ} \mathrm{C}$ for $3-4$ weeks. Glia were not removed from the cultures. For cellular ATP levels, cells were plated into 96-well plates, and ATP levels were measured at DIV 14-16. For cell death, cells were plated into 12-well plates and LDH was measured at DIV 21-22. To measure neurite outgrowth, cells were plated into 6-well plates with cover glass, and fixed for immunocytochemistry (ICC) with anti-TH+ antibody (Pel Freez Biologicals, p40101-150) as described previously $^{55}$. Images were captured using a Zeiss inverted fluorescent microscope. The number of neurites extending from the soma were counted and the longest extent of dendrites from tip to tip on opposite sides of the soma at $180^{\circ}$ were measured using AxioVision 4.8. Numbers of bifurcations (within $100 \mu \mathrm{m}$ radius of the soma) were counted in Image $\mathrm{J}^{55}$. For mitochondrial membrane potential, cortical cultures were plated into 6-well plates containing cover glasses, and confocal images were captured at DIV 21 after TMRM staining.

\section{ATP synthase activity}

ATP synthase activity was measured using the ATP synthase Specific Activity Microplate Assay Kit (abcam, ab109716). ATP Synthase is immunocaptured within the wells. ATP hydrolysis results in production of ADP which is ultimately coupled to the oxidation of NADH to NAD+ that is then monitored as a decrease in absorbance at $340 \mathrm{~nm}$. Fresh rat SMVs were adjusted to concentration $5.5 \mathrm{mg} / \mathrm{mL}$, and then extracted in detergent buffer (provided with the kit) on ice for $30 \mathrm{~min}$ followed by centrifugation at $16,000 \mathrm{rpm}$ for $20 \mathrm{~min}$. Micro plates with $5 \mu \mathrm{l}$ SMVs per well were kept at $4{ }^{\circ} \mathrm{C}$ overnight. Each well was rinsed twice with solution 1 and incubated with lipid mix for 40 min. An equal amount of WT DJ-1 or mutant DJ-1 protein was added into each reaction, and incubated for another $5 \mathrm{~min}$. Reagent mix was added to the reaction and ATP synthase activity was measured at OD340 at $1 \mathrm{~min}$ intervals for $1-2 \mathrm{~h}$.

\section{Electrophysiology}

$\mathrm{F}_{1} \mathrm{~F}_{\mathrm{O}}$ ATPase vesicle (SMV) recordings were made by forming a giga-ohm seal onto SMVs in intracellular solution $(120 \mathrm{mM} \mathrm{KCl}, 8 \mathrm{mM} \mathrm{NaCl}, 0.5 \mathrm{mM}$ EGTA, and $10 \mathrm{mM}$ HEPES, at $\mathrm{pH}$ 7.3) using an Axopatch 200B amplifier (Axon Instruments) at room temperature $\left(22-25^{\circ} \mathrm{C}\right)$. Recording electrodes were pulled from borosilicate glass capillaries (World Precision Instruments) with a final resistance in the range of 80-120 MW. SMVs were visualized by phase-contrast microscopy with a Nikon or Zeiss inverted microscope. Signals were filtered at $5 \mathrm{kHz}$ using the amplifier circuitry. Data were analyzed using pClamp 10.0 software (Axon Instruments). All population data were expressed as mean \pm SEM. Membrane currents under different experimental conditions were assessed by measuring the peak membrane current (in pico-amperes) minus the baseline current. The baseline current was defined as a nonspecific electrode leak current. All current measurements were adjusted for the holding voltage assuming a linear current-voltage relationship: the resulting conductances are expressed in picosiemens according to the equation $G=V / \Delta \mathrm{I}$, where $G$ is the conductance in picosiemens, $V$ is the membrane holding voltage in millivolts, and $\Delta \mathrm{I}$ is the peak membrane current in pico-amperes minus the baseline current in pico-amperes. Group data were quantified in terms of conductance.

\section{Mitochondrial membrane potential}

Three-week-old WT and DJ-1 ${ }^{-1-}$ cortical neurons were incubated in growth medium containing $5 \mathrm{nM}$ TMRM (tetramethylrhodamine, methyl ester, a cell membrane permeable mitochondrial potential indicator) for $20 \mathrm{~min}$ at $37^{\circ} \mathrm{C} \mathrm{CO} \mathrm{CO}_{2}$ incubator. Images were captured under standard conditions using Zeiss LSM 710 Duo confocal and the staining intensity data were analyzed using Zen software.

\section{Oxygen flux measurements}

Oxygen uptake was measured in single neurons in culture in extracellular recording buffer using a sensitive oxygen electrode. Oxygen flux was recorded using a 2-4$\mu \mathrm{m}$-diameter electrode placed at $5 \mu \mathrm{m}$ from the cell that was moved with a displacement of $10 \mu \mathrm{m}$ in the $x-y$ axes so that the oxygen-sensing electrode was positioned repeatedly closer to and farther from the cell. Signal extraction is performed by comparing near pole and far pole signal amplitudes obtained by excursion of the electrode repeatedly between the two poles (self-referencing configuration). In this manner, the effect of background, noise and drift are significantly reduced. The current detected at the two positions results in a differential current that is translated into $\mathrm{O}_{2}$ flux given the $\mathrm{O}_{2}$ concentration in the bath, the diffusion constant, and the 
distance of the excursion of the electrode between the two points.

\section{Quantitative real time RT-PCR}

Total RNA was extracted from the midbrain culture neurons using RNeasy Plus Mini Kit according to the manufacturer's protocol (Qiagen). Total RNA $(1 \mu \mathrm{g})$ was reverse transcribed using Bio-Rad iScript first cDNA synthesis kit. Quantitation of mRNA levels was performed by TaqMan Gene Expression Assays (Thermo Fisher Scientific, USA). Analyses were calculated using the $2^{-\Delta \Delta C T}$ method with $\beta$-actin as the normalizing endogenous control. The following probes were used:

\begin{tabular}{ll}
\hline Name & Assay/Probe ID \\
\hline hACTB & Hs01060665_g1 \\
hATP5G1 & Hs00829069_s1 \\
hATP5G2 & Hs01086654_g1 \\
hATP5G3 & Hs00909659_m1 \\
hATP5B & Hs00969569_m1 \\
hATP5O & Hs00426889_m1 \\
mATP5G1 & Mm02601566_g1 \\
mATP5G2 & Mm00848143_g1 \\
mATP5G3 & Mm01334541_g1 \\
mActB & Mm02619580_g1 \\
mATP5B & Mm01160389_g1
\end{tabular}

\section{Statistical analyses}

Data in graphs were shown as mean \pm SEM. For two group comparisons, paired or unpaired Student's $t$-tests (two-tailed) were used. For multiple comparisons, statistical analyses were performed with ANOVA. In some cases, data were normalized to control data before analysis ( $P<0.05$; ${ }^{* *} P<0.01$; ${ }^{* * *} P<0.001$; $\left.{ }^{* * * *} P<0.0001\right)$.

\section{Acknowledgements}

The research was supported by NIH (NS081746) to E.A.J., W.M. and V.B. are supported by the Stichting Parkinson Fonds (The Netherlands).

\footnotetext{
Author details

${ }^{1}$ Department of Internal Medicine (Endocrinology), Yale University, New Haven, CT, USA. ${ }^{2}$ Department of Human Nutrition and Hospitality Management, University of Alabama, Tuscaloosa, AL, USA. ${ }^{3}$ Department of Cell Biology, Yale University, New Haven, CT, USA. ${ }^{4}$ Department of Neurology, Erasmus MC, Rotterdam, The Netherlands. ${ }^{5}$ Neurology Service and Stroke Unit, Brotzu General Hospital, Cagliari, Italy. ${ }^{6}$ Department of Clinical Genetics, Erasmus MC, Rotterdam, The Netherlands. 'Institute of Life Sciences, University of Southampton, Southampton, England. ${ }^{8}$ Marine Biological Laboratory, Woods Hole, MA, USA. ${ }^{\circ}$ Division of Brain Sciences, Department of Medicine, Imperial College, London, UK. ${ }^{10}$ Department of Neuroscience, Yale University, New Haven, CT, USA
}

\section{Conflict of interest}

V.B. received research grants from the Stichting Parkinson Fonds (the Netherlands); the ZonMw (the Netherlands), under the aegis of the EU Joint Program-Neurodegenerative Disease Research (JPND); the Centre for Human Drug Research (Leiden, the Netherlands); and the Erasmus MC, Rotterdam. He receives compensation for serving as Section Editor of Current Neurology and Neuroscience Reports, and Editor-in-Chief of Parkinsonism and related disorders. He received honoraria from the International Parkinson and Movement Disorder Society, the Centre for Human Drug Research (the Netherlands), and the Sun Pharmaceutical Laboratories Limited (India). W.M. received research grants from the Stichting Parkinson Fonds (the Netherlands). E.A.J. receives compensation for serving as Associate Editor for the FASEB Journal. The other authors declare that they have no conflict of interest.

\section{Publisher's note}

Springer Nature remains neutral with regard to jurisdictional claims in published maps and institutional affiliations.

Supplementary Information accompanies this paper at (https://doi.org/ 10.1038/s41419-019-1679-x).

Received: 21 January 2019 Revised: 10 April 2019 Accepted: 19 May 2019 Published online: 13 June 2019

\section{References}

1. Albers, D. S. \& Beal, M. F. Mitochondrial dysfunction and oxidative stress in aging and neurodegenerative disease. J. Neural Transm. Suppl. 59, 133-154 (2000).

2. Banerjee, R., Starkov, A. A., Beal, M. F. \& Thomas, B. Mitochondrial dysfunction in the limelight of Parkinson's disease pathogenesis. Biochim. Biophys. Acta 1792, 651-663 (2009).

3. Beal, M. F. Mitochondria take center stage in aging and neurodegeneration. Ann. Neurol. 58, 495-505 (2005).

4. Vicario, M., Cieri, D., Brini, M. \& Cali, T. The close encounter between alphasynuclein and mitochondria. Front. Neurosci. 12, 388 (2018).

5. Giguere, N. et al. Comparative analysis of Parkinson's disease-associated genes in mice reveals altered survival and bioenergetics of Parkin-deficient dopamine neurons. J. Biol. Chem. 293, 9580-9593 (2018).

6. Chu, C. T. Multiple pathways for mitophagy: a neurodegenerative conundrum for Parkinsona's disease. Neurosci. Lett. 697, 66-71 (2019).

7. Reed, X., Bandres-Ciga, S., Blauwendraat, C. \& Cookson, M. R. The role of monogenic genes in idiopathic Parkinson's disease. Neurobiol. Dis. 124, 230-239 (2018).

8. Bonifati, V. et al. Mutations in the DJ-1 gene associated with autosomal recessive early-onset parkinsonism. Science 299, 256-259 (2003).

9. Kim, R. H. et al. Hypersensitivity of DJ-1-deficient mice to 1-methyl-4-phenyl1,2,3,6-tetrahydropyrindine (MPTP) and oxidative stress. Proc. Natl Acad. Sci. USA 102, 5215-5220 (2005).

10. Biosa, A. et al. Recent findings on the physiological function of DJ-1: beyond Parkinson's disease. Neurobiol. Dis. 108, 65-72 (2017).

11. Hao, L. Y., Giasson, B. I. \& Bonini, N. M. DJ-1 is critical for mitochondrial function and rescues PINK1 loss of function. Proc. Natl Acad. Sci. USA 107, 9747-9752 (2010).

12. Malgieri, G. \& Eliezer, D. Structural effects of Parkinson's disease linked DJ-1 mutations. Protein Sci. 17, 855-868 (2008).

13. Larsen, N. J., Ambrosi, G., Mullett, S. J., Berman, S. B. \& Hinkle, D. A. DJ-1 knockdown impairs astrocyte mitochondrial function. Neuroscience 196, 251-264 (2011).

14. Junn, E., Jang, W. H., Zhao, X., Jeong, B. S. \& Mouradian, M. M. Mitochondrial localization of DJ-1 leads to enhanced neuroprotection. J. Neurosci. Res. 87, 123-129 (2009).

15. Canet-Aviles, R. M. et al. The Parkinson's disease protein DJ-1 is neuroprotective due to cysteine-sulfinic acid-driven mitochondrial localization. Proc Natl Acad. Sci. USA 101, 9103-9108 (2004).

16. Giaime, E., Yamaguchi, H., Gautier, C. A., Kitada, T. \& Shen, J. Loss of DJ-1 does not affect mitochondrial respiration but increases ROS production and mitochondrial permeability transition pore opening. PLOS ONE 7, e40501 (2012). 
17. Alavian, K. N. et al. An uncoupling channel within the c-subunit ring of the F1FO ATP synthase is the mitochondrial permeability transition pore. Proc. Natl Acad. Sci. USA 111, 10580-10585 (2014).

18. Bonora, $\mathrm{M}$. et al. Role of the $\mathrm{c}$ subunit of the FO ATP synthase in mitochondrial permeability transition. Cell Cycle 12, 674-683 (2013).

19. Beutner, G., Alanzalon, R. E. \& Porter, G. A. Jr. Cyclophilin D regulates the dynamic assembly of mitochondrial ATP synthase into synthasomes. Sci. Rep. 7, 14488 (2017).

20. Giorgio, V. et al. Dimers of mitochondrial ATP synthase form the permeability transition pore. Proc. Natl Acad. Sci. USA 110, 5887-5892 (2013).

21. Azarashvili, T. et al. Potential role of subunit c of FOF1-ATPase and subunit c of storage body in the mitochondrial permeability transition. Effect of the phosphorylation status of subunit c on pore opening. Cell Calcium 55, 69-77 (2014).

22. Ludtmann, M. H. R. et al. Alpha-synuclein oligomers interact with ATP synthase and open the permeability transition pore in Parkinson's disease. Nat. Commun. 9, 2293 (2018).

23. Giorgio, V. et al. Cyclophilin D modulates mitochondrial FOF1-ATP synthase by interacting with the lateral stalk of the complex. J. Biol. Chem. 284, 33982-33988 (2009)

24. Alavian, K. N. et al. Bcl-xL regulates metabolic efficiency of neurons through interaction with the mitochondrial F1FO ATP synthase. Nat. Cell Biol. 13, 1224-1233 (2011).

25. Alavian, K. N. et al. The mitochondrial complex v-associated large-conductance inner membrane current is regulated by cyclosporine and dexpramipexole. Mol. Pharmacol. 87, 1-8 (2015).

26. Chen, Y. B. et al. BCl-XL regulates mitochondrial energetics by stabilizing the inner membrane potential. J. Cell Biol. 195, 263-276 (2011).

27. Chen, C. et al. Mitochondrial ATP synthasome: three-dimensional structure by electron microscopy of the ATP synthase in complex formation with carriers for Pi and ADP/ATP. J. Biol. Chem. 279, 31761-31768 (2004).

28. Ko, Y. H., Delannoy, M., Hullihen, J., Chiu, W. \& Pedersen, P. L. Mitochondrial ATP synthasome. Cristae-enriched membranes and a multiwell detergent screening assay yield dispersed single complexes containing the ATP synthase and carriers for Pi and ADP/ATP. J. Biol. Chem. 278, 12305-12309 (2003).

29. Caviston, T. L., Ketchum, C. J., Sorgen, P. L., Nakamoto, R. K. \& Cain, B. D. Identification of an uncoupling mutation affecting the $b$ subunit of F1F0 ATP synthase in Escherichia coli. FEBS Lett. 429, 201-206 (1998).

30. Land, S. C., Porterfield, D. M., Sanger, R. H. \& Smith, P. J. The self-referencing oxygen-selective microelectrode: detection of transmembrane oxygen flux from single cells. J. Exp. Biol. 202, 211-218 (1999).

31. Smith, P. J. S., Sanger, R. H. \& Messerli, M. A. in Electrochemical methods for neuroscience frontiers in neuroengineering (eds Michael A. C. \& Borland L. M.) (CRC Press/Taylor \& Francis, Boca Raton (FL), 2007).

32. Smith, P. J. S., Sanger, R. S. \& Messerli, M. A. Principles, development and applications of self-referencing electrochemical microelectrodes to the determination of fluxes at cell membranes. In: Methods and new frontiers in neuroscience (eds Michael, A.C.) Ch. 18 (CRC Press, 2007).

33. Alavian, K. N. et al. Effects of dexpramipexole on brain mitochondrial conductances and cellular bioenergetic efficiency. Brain Res. S0006-8993(12)00128X [pii] https://doi.org/10.1016/j.brainres.2012.01.046 (2012).

34. Cudkowicz, M. et al. The effects of dexpramipexole (KNS-760704) in individuals with amyotrophic lateral sclerosis. Nat. Med. 17, 1652-1656 (2011).
35. Jonas, E. A., Porter, G. A. \& Alavian, K. N. BCl-xL in neuroprotection and plasticity. Front. Physiol. 5, 355 (2014).

36. Guzman, J. N. et al. Oxidant stress evoked by pacemaking in dopaminergic neurons is attenuated by DJ-1. Nature 468, 696-700 (2010).

37. Taira, T. et al. DJ-1 has a role in antioxidative stress to prevent cell death. EMBO Rep. 5, 213-218 (2004)

38. Yamane, $\mathrm{T}$. et al. Expression and protease activity of mouse legumain are regulated by the oncogene/transcription co-activator, DJ-1 through p53 and cleavage of annexin A2 is increased in DJ-1-knockout cells. Biochem. Biophys. Res. Commun. 467, 472-477 (2015).

39. Yamaguchi, S. et al. Transcriptional activation of low-density lipoprotein receptor gene by DJ-1 and effect of DJ-1 on cholesterol homeostasis. PLOS ONE 7, e38144 (2012).

40. Kim, S. J. et al. Nuclear translocation of DJ-1 during oxidative stress-induced neuronal cell death. Free Radic. Biol. Med. 53, 936-950 (2012).

41. Batelli, S. et al. DJ-1 modulates alpha-synuclein aggregation state in a cellular model of oxidative stress: relevance for Parkinson's disease and involvement of HSP70. PLOS ONE 3, e1884 (2008).

42. Li, H. M., Niki, T., Taira, T., Iguchi-Ariga, S. M. \& Ariga, H. Association of DJ-1 with chaperones and enhanced association and colocalization with mitochondrial Hsp70 by oxidative stress. Free Radic. Res. 39, 1091-1099 (2005).

43. De Miranda, B. R. et al. Astrocyte-specific DJ-1 overexpression protects against rotenone-induced neurotoxicity in a rat model of Parkinson's disease. Neurobiol. Dis. 115, 101-114 (2018).

44. $\mathrm{Xu}, \mathrm{C}$. Y. et al. DJ-1 Inhibits alpha-synuclein aggregation by regulating chaperone-mediated autophagy. Front. Aging Neurosci. 9, 308 (2017).

45. Beck, S. J. et al. Deregulation of mitochondrial F1FO-ATP synthase via OSCP in Alzheimer's disease. Nat. Commun. 7, 11483 (2016).

46. $\mathrm{Li}, \mathrm{H}$. et al. A BCl-xL-Drp1 complex regulates synaptic vesicle membrane dynamics during endocytosis. Nat. Cell Biol. 15, 773-785 (2013).

47. $\mathrm{Li}, \mathrm{H}$. et al. BCl-XL induces Drp1-dependent synapse formation in cultured hippocampal neurons. Proc. Natl Acad. Sci. USA 105, 2169-2174 (2008).

48. Berman, S. B. et al. BCl-x L increases mitochondrial fission, fusion, and biomass in neurons. J. Cell Biol. 184, 707-719 (2009).

49. Courchet, J. et al. Terminal axon branching is regulated by the LKB1-NUAK1 kinase pathway via presynaptic mitochondrial capture. Cell 153, 1510-1525 (2013).

50. Irrcher, I. et al. Loss of the Parkinson's disease-linked gene DJ-1 perturbs mitochondrial dynamics. Hum. Mol. Genet. 19, 3734-3746 (2010).

51. Giguere, N. et al. Comparative analysis of Parkinson's disease-associated genes reveals altered survival and bioenergetics of parkin-deficient dopamine neurons in mice. J. Biol. Chem. https://doi.org/10.1074/jbc.RA117.000499 (2018).

52. Rousseaux, M. W. et al. Progressive dopaminergic cell loss with unilateral-tobilateral progression in a genetic model of Parkinson disease. Proc. Natl Acad. Sci. USA 109, 15918-15923 (2012).

53. Zuo, J., Lei, M., Wen, M., Chen, Y. \& Liu, Z. Overexpression of ATP5b promotes cell proliferation in asthma. Mol. Med. Rep. 16, 6946-6952 (2017).

54. Goldberg, M. S. et al. Nigrostriatal dopaminergic deficits and hypokinesia caused by inactivation of the familial Parkinsonism-linked gene DJ-1. Neuron 45, 489-496 (2005)

55. Park, H. A., Licznerski, P., Alavian, K. N., Shanabrough, M. \& Jonas, E. A. BCl-xL is necessary for neurite outgrowth in hippocampal neurons. Antioxid. Redox Signal. https://doi.org/10.1089/ars.2013.5570 (2014). 